\title{
Wavevector Selective Metasurfaces and Tunnel Vision Filters
}

\author{
Vassili A Fedotov ${ }^{1}$, Jan Wallauer ${ }^{2}$, Markus Walther ${ }^{2}$, Mauro Perino ${ }^{1,3}$, Nikitas Papasimakis ${ }^{1}$ and \\ Nikolay I Zheludev ${ }^{1,4}$
}

Metasurfaces offer unprecedented flexibility in the design and control of light propagation, replacing bulk optical components and exhibiting exotic optical effects. One of the basic properties of the metasurfaces, which renders them as frequency selective surfaces, is the ability to transmit or reflect radiation within a narrow frequency band that can be engineered on demand. Here we introduce and demonstrate experimentally in the $\mathrm{THz}$ domain the concept of wavevector selective surfaces - metasurfaces transparent only within a narrow range of light propagation directions operating effectively as tunnel vision filters. Practical implementations of the new concept include applications in wavefront manipulation, observational instruments, vision and free-space communication in light-scattering environments.

Light: Science \& Applications (2015) 4, e306; doi:10.1038/lsa.2015.79; published online 3 July 2015

Keywords: flat optics; metafilms; metasurfaces; planar metamaterials; wavefront manipulation

\section{INTRODUCTION}

Metasurfaces (also known as planar metamaterials or metafilms) are a special low-dimensional class of artificially structured media. This class is represented by thin metal films and surfaces periodically patterned on a sub-wavelength scale, which can be readily fabricated using the existing planar technologies. Apart from their spectral selectivity ${ }^{1}$ metasurfaces have demonstrated intriguing electromagnetic effects such as asymmetric transmission ${ }^{2,3}$ and optical activity without structural chirality ${ }^{4}$. They can exhibit resonant dispersion mimicking electromagnetically induced transparency and the slow-light phenomenon $^{5-7}$, be invisible ${ }^{8}$, efficiently convert polarization ${ }^{9-11}$, or perfectly absorb radiation ${ }^{12,13}$. Metasurfaces with gradient structuring anomalously reflect and refract light ${ }^{10-15}$ and can act as lenses, wave-plates, and diffraction gratings ${ }^{16-19}$. Planar metamaterials are also able to enhance the light-matter interaction facilitating sensing ${ }^{20}$, energy harvesting $^{21}$, and generation of coherent radiation ${ }^{22,23}$.

The functionality of the most common types of planar metamaterials is determined by the individual resonant response of their basic structural elements - metamolecules, which are only weakly coupled to each other. When electromagnetic coupling between the metamolecules is strong ${ }^{24}$ the relative phase of their excitation becomes important and the resulting spectral response is no longer determined by the individual resonances of the metamolecules. The metamaterial spectrum is then shaped by the collective, spatially coherent modes of metamolecular excitations that engage a large ensemble of metamolecules ${ }^{25}$. The introduction of structural disorder in such an ensemble reduces the degree of coherency and leads to the weakening and broadening of its collective resonant response, which might be seen to vanish at even moderate levels of the disorder ${ }^{24,26}$. Strong inter-metamolecular coupling is also responsible for the 'size effect' where the resonant transmission band of a metamaterial narrows with increasing size of the metamaterial sample ${ }^{27}$.

In this paper we show that strong inter-metamolecular coupling can lead to a new phenomenon of 'tunnel vision', which renders a coherent metasurface transparent within a very narrow range of light propagation directions. This effect is accompanied by 'rectification' of incident wavefronts, when initially spherical waves emerge as planar while traversing such a metasurface in the absence of any spatial phase modulation or adaptive feedback ${ }^{28}$. The transmitted wavefronts appear parallel to the plane of the metamaterial and the effect does not depend on the curvature of the incident wavefronts.

As illustrated in Figure 1, such response is fundamentally different from that of conventional convex lenses and recently demonstrated metasurface-based lenses ${ }^{16,19}$. Indeed, a lens introduces a spatially dependent delay in the optical path, which compensates the curvature of the incident spherical wavefront if its source is located in the 'focal spot' of the lens. In a convex glass lens, for example, the spatial variation of optical delay is achieved by gradually reducing the thickness of the lens towards its edges (see Figure 1a). In a metasurface-based lens the same is accomplished by changing the size and/or shape of its metamolecules: the resulting position-dependent phase lag for light scattered by the metamolecules mimics spatial variation of optical delay in the glass lens (see Figure 1b). For spherical waves originating at its focal point, the lens converts the entire range of incident wavevectors into a much narrower range that converges around the optical axis of the lens.

${ }^{1}$ Optoelectronics Research Centre and Centre for Photonic Metamaterials, University of Southampton, Southampton, SO17 1BJ, UK; ${ }^{2}$ Department of Molecular and Optical Physics, University of Freiburg, D-79104 Freiburg, Germany; ${ }^{3}$ Department of Information Engineering, University of Padova, Padova, Italy and ${ }^{4}$ Centre for Disruptive Photonic Technologies, TPI, Nanyang Technological University, 637371 Singapore, Singapore. 
a

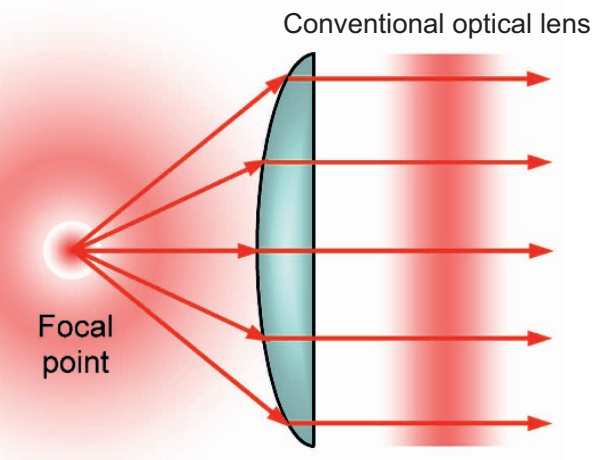

b

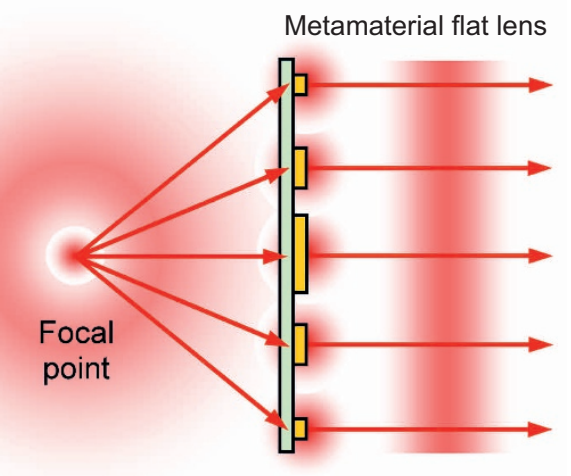

c

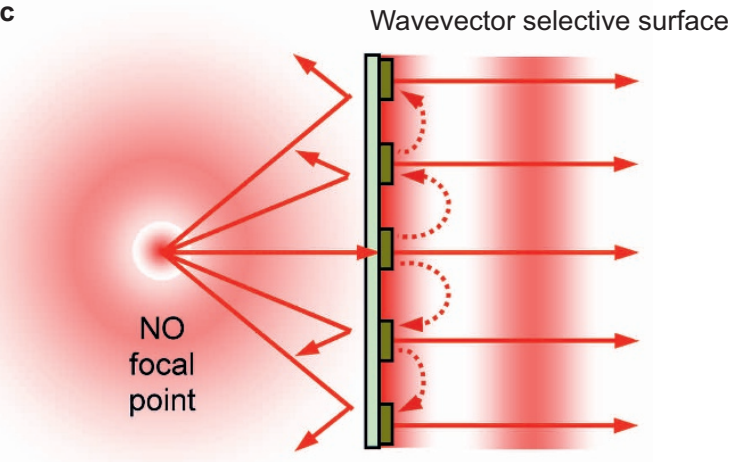

Figure 1. Three ways of producing planar wavefronts with a point light source.

The 'tunnel vision' effect can be understood as wavevector filtering, which occurs in a narrow transparency window of the metasurface corresponding to the collective resonance of its strongly coupled metamolecules. Indeed, in a regular planar array of identical metamolecules with the sub-wavelength period $d$ the coupling is strongest when the metamolecules are excited by a normally incident plane wave, i.e. they all oscillate in-phase. Any plane wave with its $k$-vector deviating from the array's normal (so that $k_{\|} \neq 0$ ) introduces a phase delay in the excitation of the metamolecules that linearly varies along the metasurface. The resulting de-phasing reduces the strength of coupling through the factor $\cos \left(k_{\|} d\right)$ and hence changes the energy of the collective mode (see Supplementary Information). Consequently, the transparency window shifts to a different frequency and the metasurface becomes opaque. Operating at the frequency of its collective resonance the structure therefore acts as a wavevector selective surface (WSS): it admits only plane waves with $k$-vectors parallel (or nearly parallel) to its normal, while all other waves are reflected back and/or absorbed (see Figure 1c).

\section{MATERIALS AND METHODS}

\section{Modeling}

The propagation of spherical wavefronts through a wavevector selective surface was simulated using a 3D Maxwell's equations solver of the COMSOL Multiphysics simulation package 3.5a (COMSOL, Inc., Burlington, USA). The metasurface was modeled as a $14 \times 14$ array of asymmetrically split rings (ASRs) with the period $d=565 \mu \mathrm{m}$. The rings had the radius $r=245 \mu \mathrm{m}$ and were split into wire sections $140^{\circ}$ and $160^{\circ}$ long separated by equal gaps. The wire sections had the width of $40 \mu \mathrm{m}$ and were modeled as perfect electric conductors. The thickness of the supporting substrate was $120 \mu \mathrm{m}$ and its permittivity was assumed to be $\varepsilon=2.2$. The size of the simulation domain allocated for the transmitted wavefronts was $7910 \times 7910 \times 7910 \mu \mathrm{m}$.

\section{Experiment}

The metamaterial sample had been fabricated by etching a $9-\mu \mathrm{m}$-thick copper layer covering one side of a 120 - $\mu \mathrm{m}$-thick low-loss teflon substrate and closely resembled the modeled split-ring array both in terms of its size and design parameters. The transmitted wavefronts were visualized in the $0.12-0.23 \mathrm{THz}$ range of frequencies using a state-ofthe-art THz-field imaging technique ${ }^{29}$, which enabled mapping of the electric-field component of the propagating waves with the spatial resolution of $160 \mu \mathrm{m}$ and frequency resolution of $0.01 \mathrm{THz}$. The spherical waves were produced by illuminating a pinhole placed $500 \mu \mathrm{m}$ away from the sample with a focused $\mathrm{THz}$ beam polarized parallel to the split of the rings. The diameter of the pinhole was $1750 \mu \mathrm{m}$, which ensured adequate signal-to-noise ratio for the wavefront imaging at low frequencies with signs of diffraction visible only above $\sim 0.2 \mathrm{THz}$.

\section{RESULTS AND DISCUSSION}

As an example of WSS we consider here a planar metamaterial based on ASRs, a regular array of identical metamolecules formed by pairs of metallic arcs of different length (see inset to Figure 2a). For normally incident plane waves with the E-field being parallel to the split its transmission spectrum features a narrow asymmetric pass-band with a sharp roll-off (Fano resonance), which in our case is centered around $v_{0}=0.165 \mathrm{THz}$ (see Figure $2 \mathrm{a}$ ). This spectral feature corresponds to the resonant excitation of anti-symmetric charge-current mode, the so-called trapped or sub-radiant mode ${ }^{30,31}$, where charges $q$ and currents $\mathrm{j}$ induced in the opposite arcs of each ASR-metamolecule oscillate with equal amplitudes but opposite phases (see inset to Figure 2a). Such mode can be represented by a combination of an oscillating magnetic dipole with its moment being orthogonal to the metamaterial plane $m=(0,0$, $\left.m_{z}\right)$, and an electric quadrupole $Q$ characterized by two non-zero inplane components $Q_{x y}=Q_{y x}$ Being arranged in a 2D-lattice with a subwavelength unit cell these multipoles cannot contribute to the far-field scattering of the array when they oscillate in-phase: coherent superposition of their fields results in electromagnetic modes with the characteristic wavenumbers larger than $2 \pi / \lambda_{0}$, and thus the multipole radiation by the array is trapped in the near-field zone. The absence of scattering in the far-field zone renders the metamaterial transparent, while the accumulation of energy in the spatially coherent surface waves ensures strong inter-metamolecular coupling ${ }^{27}$.

For the ASR-metasurface, the effect of wavevector filtering is particularly pronounced near $0.165 \mathrm{THz}$, at the sharp edge of its transparency band where a small blue shift of the band translates into a 


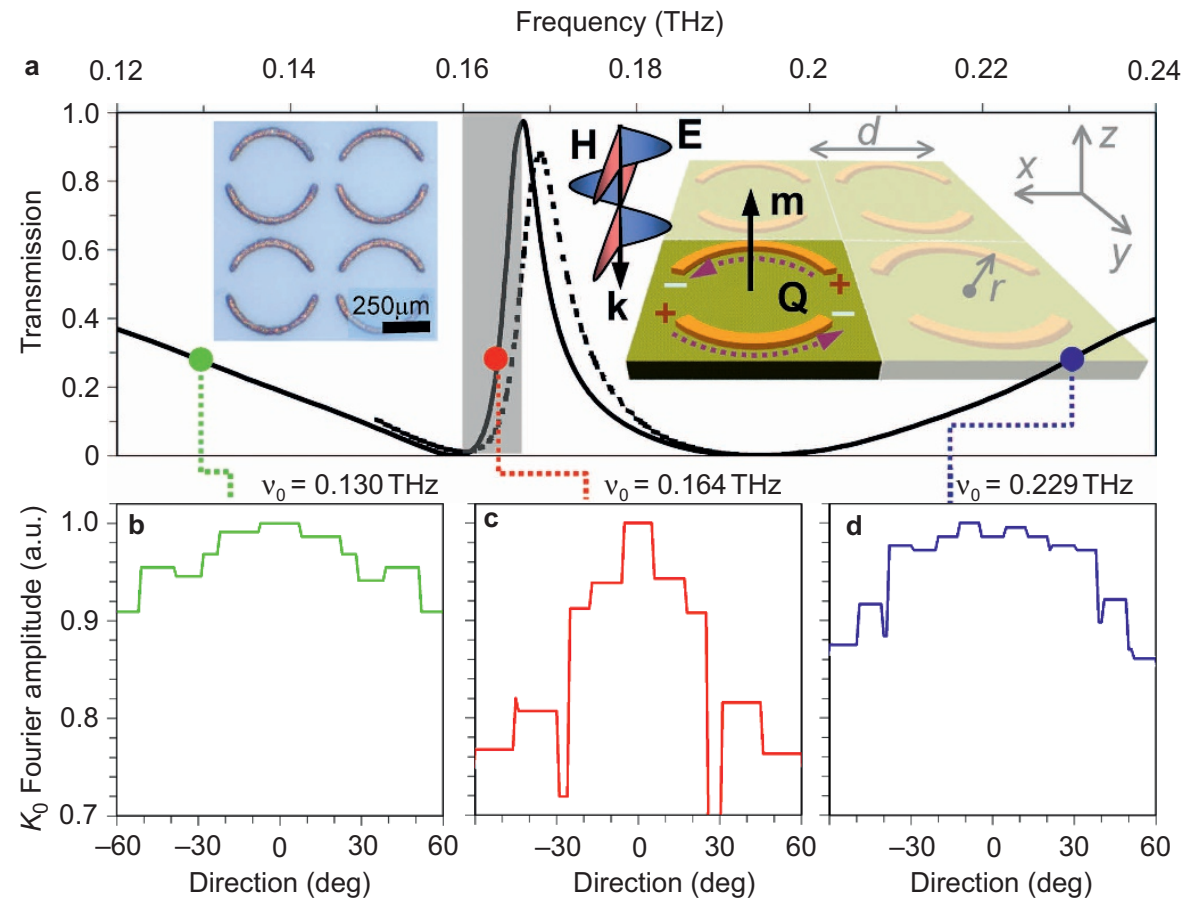

Figure 2. THz wavevector selective surface. (a) WSS transmission spectrum calculated for plane-wave illumination at normal incidence (solid curve), and $20^{\circ}$ incidence with TM polarization (dashed curve). Gray shading indicates the slope of the trapped-mode resonance. Right inset shows a schematic of the WSS, a planar array of ASRs; left inset shows a fragment of the fabricated WSS sample. (b), (c) \& (d) Angular spectra of the transmitted wavevectors calculated for spherical-wave illumination at $0.130,0.164$, and $0.229 \mathrm{THz}$, respectively.

strong reduction of the metamaterial transmission (see Figure 2a). Such a shift corresponds to an increase of the trapped-mode's energy and therefore should result from the weakening of attractive intermetamolecular coupling. In the array of ASR-metamolecules this coupling is mediated by electric quadrupole-quadrupole interaction, and at oblique incidence it is affected by the TM-component of the plane wave (see Supplementary Information).

We first demonstrated the effect of wavevector filtering numerically, by simulating the propagation of spherical waves with a large wavefront curvature through the ASR-metasurface (see Figure 3a$3 \mathrm{c}$ ). The waves were produced by an electromagnetic point source placed close to the metasurface, at a distance equal to just one period of the ASR-array, $d$, and polarized parallel to the split of the rings. The transmitted waves are visualized through the spatial variation of their phase and are presented for three characteristic frequencies $v_{0}$ (as indicated in Figure 2a), which correspond to identical levels of transmission at the edges of the stop-band $\left(v_{0}=0.130 \mathrm{THz}\right.$ and $0.229 \mathrm{THz})$ and at the pass-band $\left(v_{0}=0.164 \mathrm{THz}\right)$. As evident from Figure $3 \mathrm{a}$ and $3 \mathrm{c}$, the curvature of the wavefronts at 0.130 and $0.229 \mathrm{THz}$ remains practically unperturbed upon propagation through the metasurface. The situation changes markedly at the trapped-mode resonance, where the transmitted wavefront emerges nearly planar signifying thus the regime of wavevector filtering (see Figure $3 \mathrm{~b})$.

To characterize the response of the ASR-metasurface in terms of the transmitted $k$-vectors, i.e. partial plane-wave components, we plotted the spatial-frequency spectrum of the transmitted wavefront (see Figure 3d-3f). A perfectly spherical wave would have been represented by a circle of radius $k_{0}=2 \pi v_{0} / c$ corresponding to the fundamental spatial frequency, as indicated by the doted circles in Figure $3 \mathrm{~d}-3 \mathrm{f}$. Clearly, at $v_{0}=0.130 \mathrm{THz}$ and $v_{0}=0.229 \mathrm{THz}$ the spatial-frequency spectra have the form of partial concentric circles. The inner circle has a radius of $k_{0}$ and corresponds to the fundamental spatial frequency of the wavefront pattern. The outer circles represent higher harmonics $2 k_{0}, 3 k_{0}$ etc. that are present due to unharmonic ('saw'-like) phase variations between 0 and $2 \pi$. Given the extent of the circular patterns, the angles of incidence for the admitted partial plane-wave components fall in the relatively wide range from $-60^{\circ}$ to $+60^{\circ}$, which in our case is limited by the size of the modeled array. The amplitudes of the partial waves and therefore their relative contributions to the resulting wavefronts show little variation with the incidence angle, as evident from the angular spectra of the $k_{0}$-component presented in Figure $2 \mathrm{~b}$ and $2 \mathrm{~d}$. Thus, the response of the metasurface off the trapped-mode resonance is only weakly sensitive to the direction of the incident wavevectors.

At $v_{0}=0.164 \mathrm{THz}$, however, the spatial-frequency spectrum collapses along the horizontal axis indicating that the transmission of the metasurface becomes $k$-dependent and most of the wavevectors deviating from the structure's normal are being rejected (see Figure $3 \mathrm{e})$. This is also illustrated in Figure 2c, which shows that at $v_{0}=0.164 \mathrm{THz}$ the angular spectrum of the transmitted $k_{0}$-component converges along the $0^{\circ}$ direction within the $\left(-25^{\circ},+25^{\circ}\right)$ range of angles. The angular selectivity of the ASR-metasurface can be improved further by reducing its structural asymmetry, since the latter would increase the strength of coupling between the split-ring metamolecules ${ }^{30}$.

The effect of wavevector filtering has been confirmed also experimentally using a WSS-metamaterial sample that closely resembled the modeled ASR-array both in terms of its size and design parameters. The obtained images of the transmitted wavefronts are presented in Figure 3g-3i. Evidently, the patterns of the wavefronts, as well as their spatial-frequency spectra plotted in Figure $3 \mathrm{j}-3 \mathrm{l}$ show a 

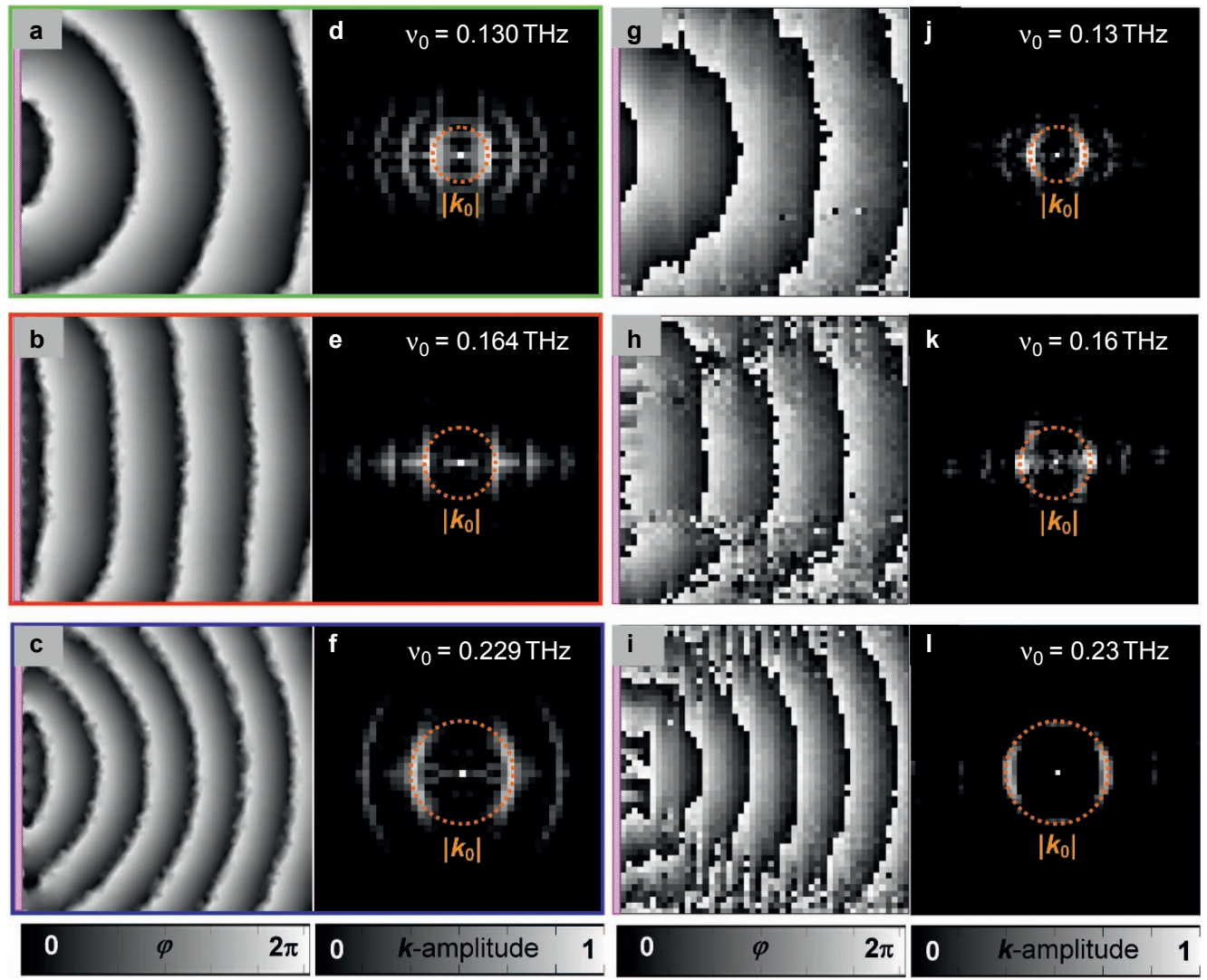

Figure 3. Wavefront transformation by ASR-based wavevector selective surface. (a-c) Modeled spatial variations of the instantaneous phase, which visualize the wavefronts transmitted by the WSS at $0.130,0.164$ and $0.229 \mathrm{THz}$ when it is illuminated by spherical waves. The waves originate from a vertically polarized point source/pinhole (not shown), on the left of the shaded purple bars, which represent the cross-section of the WSS. (d-f) Spatial-frequency spectra of the wavefronts shown in panels $(a-c)$ respectively. For clarity, the images of the spectra were enhanced by removing background noise. (g-i) Experimentally measured spatial variation of the instantaneous phase, which visualizes the wavefront of the initially spherical waves transmitted by the WSS at $0.13,0.16$, and $0.23 \mathrm{THz}$. (j-I) Spatial-frequency spectra of the wavefront patterns shown in panels (g-i), correspondingly. For clarity, the images of the spectra were enhanced by removing background noise.

very good agreement with our simulations. The appearance of noise in the phase data at $0.23 \mathrm{THz}$ (and higher frequencies) coincides with the diffraction minima due to the finite size of the pinhole aperture. Additional experimental data, including the wavefront images obtained at other frequencies and their comparison with the results of our simulations can be found in Supplementary Information. Although the limited bandwidth of the spatial-frequency filtering might be an issue for some practical applications, this problem could be addressed by employing the so-called double-continuum Fano resonance approach ${ }^{32}$, where the bandwidth of the effect is increased by stacking $2 \mathrm{D}$-chiral versions of the metamaterial (i.e. ASR structures lacking reflection symmetry $)^{33}$ with adiabatically varied resonance frequency.

Unlike the wavevector manipulation performed by the lenses, the demonstrated principle of $k$-selectivity does not rely on gradient structuring. As a result, metamaterials with strong inter-metamolecular coupling can extract plane-wave components from arbitrary shaped wavefronts. A remote analogue of WSS functionality and the associated 'tunnel vision' effect might be found in conventional rayoptics systems such as astronomical telescopes: for the same magnification the telescopes with higher $f$-ratio (i.e. slower telescopes) will allow an observer to see stars and nebulas on a much darker background yielding overall higher contrast images. Such telescopes have smaller field of view, which limits the directions of the admitted light rays to those nearly parallel to the axis of the 'tunnel' (i.e. telescope) hence blocking most of the light scattered by the atmosphere and immediate surrounding.

\section{CONCLUSIONS}

In conclusion, we have shown that strong electromagnetic coupling among the basic structural elements of a planar metamaterial provides a new degree of freedom to light manipulation, leading to an intriguing effect of wavefront rectification and tunnel vision. The effect results in arbitrary-shaped wavefronts becoming planar as they traverse the plane of the metamaterial in the absence of any spatial phase modulation or adaptive feedback, and is demonstrated here both theoretically and experimentally in the $\mathrm{THz}$ part of the spectrum. The proposed concept of wavevector selective surfaces can have a number of unique applications. For example, WSSs can improve the characteristics of observational instruments by blocking stray light and therefore acting as a flat analogue of a lens hood; or by reducing the effect of light scattering emanating from the immediate surrounding, dew, dust or scratches. WSSs can be exploited for directional filtering in free-space communications in highly turbid or strongly scattering media, yielding an improved signal-to-noise ratio. 


\section{ACKNOWLEDGEMENTS}

This work is supported by the UK's Engineering and Physical Sciences Research Council through Career Acceleration Fellowship EP/G00515X/1 (V.A.F.) and Programme grant EP/G060363/1, by the Royal Society, and by the MOE Singapore grant MOE2011-T3-1-005. Following a period of embargo, the data from this paper can be obtained from the University of Southampton ePrints research repository: http://dx.doi.org/10.5258/SOTON/376845.

1 Munk BA. Frequency Selective Surfaces: Theory and Design. New York: Wiley Interscience, 2000

2 Schwanecke AS, Fedotov VA, Khardikov VV, Prosvirnin SL, Chen Y et al. Nanostructured metal film with asymmetric optical transmission. Nano Lett 2008; 8: 2940-2943

3 Menzel C, Helgert C, Rockstuhl C, Kley E-B, Tünnermann A et al. Asymmetric transmission of linearly polarized light at optical metamaterials. Phys Rev Let 2010; 104: 253902.

4 Plum E, Liu X-X, Fedotov VA, Chen Y, Tsai DP et al. Metamaterials: optical activity without chirality. Phys Rev Lett 2009; 102: 113902.

5 Papasimakis N, Fedotov VA, Zheludev NI, Prosvirnin SL. Metamaterial analog of electromagnetically induced transparency. Phys Rev Lett 2008; 101: 253903.

6 Zhang S, Genov DA, Wang Y, Liu M, Zhang X. Plasmon-induced transparency in metamaterials. Phys Rev Lett 2008; 101: 047401

7 Tassin P, Zhang L, Koschny T, Economou EN, Soukoulis CM. Low-loss metamaterials based on classical electromagnetically induced transparency. Phys Rev Lett 2009; 102 053901.

8 Fedotov VA, Mladyonov PL, Prosvirnin SL, Zheludev NI. Planar electromagnetic metamaterial with a fish scale structure. Phys Rev E 2005; 72: 056613.

9 Hao J, Yuan Y, Ran L, Jiang T, Kong JA et al. Manipulating electromagnetic wave polarizations by anisotropic metamaterials. Phys Rev Lett 2007; 99: 063908.

10 Grady NK, Heyes JE, Chowdhury DR, Zeng Y, Reiten MT et al. Terahertz metamaterials for linear polarization conversion and anomalous refraction. Science 2013; 340 1304-1307.

11 Ma HF, Wang GZ, Kong GS, Cui TJ. Broadband circular and linear polarization conversions realized by thin birefringent reflective metasurfaces. Opt Mat Express 2014; 4: 1717-1724.

12 Fedotov VA, Prosvirnin SL, Rogacheva AV, Zheludev NI. Mirror that does not change the phase of reflected wave. Appl Phys Lett 2006; 88: 091119.

13 Landy NI, Sajuyigbe S, Mock JJ, Smith DR, Padilla WJ. Perfect metamaterial absorber. Phys Rev Lett 2008; 100: 207402.

$14 \mathrm{Yu}$ N, Genevet P, Kats MA, Aieta F, Tetienne JP et al. Light propagation with phase discontinuities: generalized laws of reflection and refraction. Science 2011; 334 333-337.

15 Ni X, Emani NK, Kildishev AV, Boltasseva A, Shalaev VM. Broadband light bending with Plasmonic Nanoantennas. Science 2012; 335: 427.
16 Tsai Y-J, Larouche S, Tyler T, Lipworth G, Jokerst NM et al. Design and fabrication of a metamaterial gradient index diffraction grating at infrared wavelengths. Opt Express 2011; 19: 24411.

17 Aieta F, Genevet P, Kats MA, Yu N, Blanchard R et al. Aberration-free ultrathin flat lenses and axicons at telecom wavelengths based on plasmonic metasurfaces. Nano Lett2012; 12: 4932-4936.

18 Ishii S, Shalaev VM, Kildishev AV. Holey-metal lenses: sieving single modes with proper phases. Nano Lett 2013; 13: 159-163.

19 Roy T, Rogers ETF, Zheludev NI. Sub-wavelength focusing meta-lens. Opt Express 2013; 21: 7577-7582.

20 Zhao J, Zhang C, Braun PV, Giessen H. Large-area low-cost plasmonic nanostructures in the NIR for Fano resonant sensing. Adv Mater 2012; 24: OP247.

21 Savinov V, Fedotov VA, de Groot PAJ, Zheludev NI. Radiation-harvesting resonan superconducting sub-THz metamaterial bolometer. Supercond Sci Technol 2013; 26: 084001.

22 Zheludev NI, Prosvirnin SL, Papasimakis N, Fedotov VA. Lasing spaser. Nature Photonics 2008; 2: 351-354.

23 Adamo G, Ou JY, So JK, Jenkins SD, de Angelis F et al. Electron-beam-driven collective-mode metamaterial light source. Phys Rev Lett 2012; 109: 217401.

24 Papasimakis N, Fedotov VA, Fu YH, Tsai DP, Zheludev NI et al. Coherent and incoherent metamaterials and order-disorder transitions. Phys Rev B 2009; 80: 041102(R).

25 Jenkins SD, Ruostekoski J. Theoretical formalism for collective electromagnetic response of discrete metamaterial systems. Phys Rev B 2012; 86: 085116.

26 Helgert C, Rockstuhl C, Etrich C, Menzel C, Kley E-B et al. Effective properties of amorphous metamaterials. Phys Rev B 2009; 79: 233107.

27 Fedotov VA, Papasimakis N, Plum E, Bitzer A, Walther M et al. Spectral collapse in ensembles of metamolecules. Phys Rev Lett 2010; 104: 223901.

28 Fedotov VA, Wallauer J, Walther M, Papasimakis N, Zheludev NI. Wavevector selective surface. Conference on Lasers and Electro-Optics (CLEO 2014), 8-13 June 2014, San Jose, CA, USA.

29 Bitzer A, Merbold H, Thoman A, Feurer T, Helm H et al. Terahertz near-field imaging of electric and magnetic resonances of a planar metamaterial. Opt Express 2009; 17: 3826-3834.

30 Fedotov VA, Rose M, Prosvirnin SL, Papasimakis N, Zheludev NI. Sharp trapped-mode resonances in planar metamaterials with a broken structural symmetry. Phys Rev Lett 2007; 99: 147401.

31 Luk'yanchuk B, Zheludev NI, Maier SA, Halas NJ, Nordlander P et al. The Fano resonance in plasmonic nanostructures and metamaterials. Nature Mater 2010; 9: 707-715.

32 Wu C, Khanikaev AB, Shvets G. Broadband slow light metamaterial based on a doublecontinuum Fano resonance. Phys Rev Lett 2011; 106: 107403.

33 Plum E, Fedotov VA, Zheludev NI. Planar metamaterial with transmission and reflection that depend on the direction of incidence. Appl Phys Lett 2009; 94: 131901.

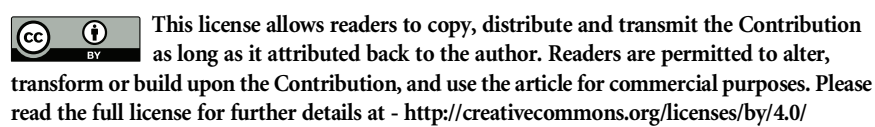

